\title{
MINIMUM PRINCIPLE AND CONTROLLABILITY FOR MULTIPARAMETER DISCRETE INCLUSIONS VIA DERIVED CONES
}

\author{
AURELIAN CERNEA
}

Received 13 December 2005; Accepted 13 February 2006

We consider a multiparameter discrete inclusion and we prove that the reachable set of a certain variational multiparameter discrete inclusion is a derived cone in the sense of Hestenes to the reachable set of the discrete inclusion. This result allows to obtain sufficient conditions for local controllability along a reference trajectory and a new proof of the minimum principle for an optimization problem given by a multiparameter discrete inclusion with endpoint constraints.

Copyright (c) 2006 Aurelian Cernea. This is an open access article distributed under the Creative Commons Attribution License, which permits unrestricted use, distribution, and reproduction in any medium, provided the original work is properly cited.

\section{Introduction}

The concept of a derived cone to an arbitrary subset of a normed space has been introduced by Hestenes in [8] and successfully used to obtain necessary optimality conditions in control theory. However, in the last years, this concept has been largely ignored in favor of other concepts of tangents cones, that may intrinsically be associated to a point of a given set: the cone of interior directions, the contingent, the quasitangent and, above all, Clarke's tangent cone.

In our previous papers [3-7], we indentified certain derived cones to the reachable sets of "ordinary" differential inclusions, hyperbolic differential inclusions, and some other classes of discrete inclusions in terms of the variational inclusion associated to the differential inclusion and to the discrete inclusion. These results allowed to obtain a simple proof of the maximum principle in optimal control and sufficient conditions for local controllability along a reference trajectory.

In the present paper, we consider a multiparameter discrete inclusion that describes the Roesser model and we prove that the reachable set of a certain variational multiparameter discrete inclusion is a derived cone in the sense of Hestenes to the reachable set of the discrete inclusion. 
As applications of our main result, we point out the possibility to obtain some refinements of the existing results in the theory of necessary optimality conditions and also in controllability theory for multiparameter discrete inclusions.

Optimal control problems for systems described by discrete inclusions have been studied by many authors $([2,10,12]$, etc. $)$. In the framework of multivalued problems, necessary optimality conditions for a problem without endpoint constraints are obtained in [10] and improved afterwards in [12]. The idea in [12] is to use a special (Warga's) open mapping theorem to obtain a sufficient condition for the discrete inclusion to be locally controllable around a given trajectory and as a consequence, via a separation result, to obtain the minimum (maximum) principle.

In contrast with the approach in [12], even if the problem studied in the present paper is more difficult, due to endpoint constraints, the method in our approach seems to be conceptually very simple, relying only on 2-3 clear-cut steps and using a minimum of auxiliary results.

The paper is organized as follows. In Section 2 we present the notations and preliminary results to be used in the sequel. Section 3 is devoted to our main result; while in Section 4 we present the above mentioned applications concerning controllability and necessary optimality conditions.

\section{Preliminaries}

For a set that is, in general, neither a differentiable manifold nor a convex set, its infinitesimal properties may be characterized only by tangent cones in a generalized sense, extending the classical concepts of tangent cones in differential geometry and convex analysis, respectively.

From the rather large number of "convex approximations," "tents," "regular tangents cones," and so forth, in the literature, we choose the concepts of a derived cone introduced by Hestenes in [8].

Definition 2.1 [8]. A subset $M \subset \mathbb{R}^{n}$ is said to be a derived set to $X \subset \mathbb{R}^{n}$ at $x \in X$ if for any finite subset $\left\{v_{1}, \ldots, v_{k}\right\} \subset M$, there exist $s_{0}>0$ and a continuous mapping $a(\cdot):\left[0, s_{0}\right]^{k} \rightarrow$ $X$ such that $a(0)=x$ and $a(\cdot)$ is (conically) differentiable at $s=0$ with the derivative $\operatorname{col}\left[v_{1}, \ldots, v_{k}\right]$ in the sense that

$$
\lim _{\mathbb{R}_{+}^{k} \ni \theta \rightarrow 0} \frac{\left\|a(\theta)-a(0)-\sum_{i=1}^{k} \theta_{i} v_{i}\right\|}{\|\theta\|}=0 .
$$

We will write in this case that the derivative of $a(\cdot)$ at $s=0$ is given by

$$
\mathrm{D} a(0) \theta=\sum_{i=1}^{k} \theta_{j} v_{j}, \quad \forall \theta=\left(\theta_{1}, \ldots, \theta_{k}\right) \in \mathbb{R}_{+}^{k}:=[0, \infty)^{k}
$$

A subset $C \subset \mathbb{R}^{n}$ is said to be a derived cone of $X$ at $x$ if it is a derived set and also a convex cone. 
For the basic properties of derived sets and cones we refer to Hestenes [8]; we recall that if $M$ is a derived set, then $M \cup\{0\}$ as well as the convex cone generated by $M$, defined by

$$
\operatorname{cco}(M)=\left\{\sum_{i=1}^{k} \lambda_{j} v_{j} ; \lambda_{j} \geq 0, k \in \mathbb{N}, v_{j} \in M, j=1, \ldots, k\right\},
$$

is also a derived set, hence a derived cone.

The fact that the derived cone is a proper generalization of the classical concepts in differential geometry and convex analysis is illustrated by the following results [8]: if $X \subset$ $\mathbb{R}^{n}$ is a differentiable manifold and $T_{X} X$ is the tangent space in the sense of differential geometry to $X$ at $x$ :

$$
T_{x} X=\left\{v \in \mathbb{R}^{n} ; \exists c:(-s, s) \longrightarrow X, \text { of class } C^{1}, c(0)=x, c^{\prime}(0)=v\right\}
$$

then $T_{x} X$ is a derived cone; also, if $X \subset \mathbb{R}^{n}$ is a convex subset, then the tangent cone in the sense of convex analysis defined by

$$
T C_{x} X=\operatorname{cl}\{t(y-x) ; t \geq 0, y \in X\}
$$

is also a derived cone. $\operatorname{By} \operatorname{cl} A$ we denote the closure of the set $A \subset \mathbb{R}^{n}$.

Since any convex subcone of a derived cone is also a derived cone, such an object may not be uniquely associated to a point $x \in X$; moreover, simple examples show that even a maximal with respect to a set-inclusion derived cone may not be uniquely defined: if the set $X \subset \mathbb{R}^{2}$ is defined by

$$
X=C_{1} \cup C_{2}, \quad C_{1}=\{(x, x), x \geq 0\}, \quad C_{2}=\{(x,-x), x \leq 0\},
$$

then $C_{1}$ and $C_{2}$ are both maximal derived cones of $X$ at the point $(0,0) \in X$.

On the other hand, the up-to-date experience in nonsmooth analysis shows that for some problems, the use of one of the intrinsic tangent cones may be preferable. From the multitude of the intrinsic tangent cones in the literature (e.g., [1]), the contingent, the quasitangent, and Clarke's tangent cones, defined, respectively, by

$$
\begin{gathered}
K_{x} X=\left\{v \in \mathbb{R}^{n} ; \exists s_{m} \longrightarrow 0+, x_{m} \in X: \frac{x_{m}-x}{s_{m}} \longrightarrow v\right\}, \\
Q_{x} X=\left\{v \in \mathbb{R}^{n} ; \forall s_{m} \longrightarrow 0+, \exists x_{m} \in X: \frac{x_{m}-x}{s_{m}} \longrightarrow v\right\}, \\
C_{x} X=\left\{v \in \mathbb{R}^{n} ; \forall\left(x_{m}, s_{m}\right) \longrightarrow(x, 0+), x_{m} \in X, \exists y_{m} \in X: \frac{y_{m}-x_{m}}{s_{m}} \longrightarrow v\right\},
\end{gathered}
$$

seem to be among the most oftenly used in the study of different problems involving nonsmooth sets and mappings. 
We recall that, in contrast with $K_{x} X, Q_{x} X$, the cone $C_{x} X$ is convex and one has $C_{x} X \subset$ $Q_{x} X \subset K_{x} X$.

It follows from Definition 2.1 and from (2.7) that if $C \subset \mathbb{R}^{n}$ is a derived cone to $X$ at $x$, then $C \subset Q_{x} X$. On the other hand, example (2.6), for which $C_{0} X=\{0\}$, shows that a derived cone may not be contained into the cone $C_{x} X$.

We recall that two cones $C_{1}, C_{2} \subset \mathbb{R}^{n}$ are said to be separable if there exists $q \in \mathbb{R}^{n} \backslash\{0\}$ such that

$$
\langle q, v\rangle \leq 0 \leq\langle q, w\rangle, \quad \forall v \in C_{1}, w \in C_{2}
$$

We denote by $C^{+}$the positive dual cone of $C \subset \mathbb{R}^{n}$ :

$$
C^{+}=\left\{q \in \mathbb{R}^{n} ;\langle q, v\rangle \geq 0, \forall v \in C\right\}
$$

The negative dual cone of $C \subset \mathbb{R}^{n}$ is $C^{-}=-C^{+}$.

The following "intersection property" of derived cones, obtained by Mirică [11], is a key tool in the proof of necessary optimality conditions.

Lemma 2.2 [11]. Let $X_{1}, X_{2} \subset \mathbb{R}^{n}$ be given sets, let $x \in X_{1} \cap X_{2}$, and let $C_{1}, C_{2}$ be derived cones to $X_{1}$, respectively, to $X_{2}$ at $x$. If $C_{1}$ and $C_{2}$ are not separable, then

$$
\operatorname{cl}\left(C_{1} \cap C_{2}\right)=\left(\operatorname{cl}\left(C_{1}\right)\right) \cap\left(\operatorname{cl}\left(C_{2}\right)\right) \subset Q_{x}\left(X_{1} \cap X_{2}\right) .
$$

For a mapping $g(\cdot): X \subset \mathbb{R}^{n} \rightarrow \mathbb{R}$ which is not differentiable, the classical (Fréchet) derivative is replaced by some generalized directional derivatives. We recall only the upper right-contingent derivatives defined by

$$
\overline{\mathrm{D}}_{K} g(x ; v)=\limsup _{(\theta, w) \rightarrow(0+, v)} \frac{g(x+\theta w)-g(x)}{\theta}, \quad v \in K_{x} X \text {, }
$$

and in the case when $g(\cdot)$ is locally-Lipschitz at $x \in \operatorname{int}(X)$ by Clarke's generalized directional derivative defined by

$$
\mathrm{D}_{C} g(x ; v)=\limsup _{(y, \theta) \rightarrow(x, 0+)} \frac{g(y+\theta v)-g(y)}{\theta}, \quad v \in \mathbb{R}^{n} .
$$

The results in the next section will be expressed in the case where $g(\cdot)$ is locallyLipschitz at $x$, in terms of the Clarke generalized gradient defined by

$$
\partial_{C} g(x)=\left\{q \in \mathbb{R}^{n} ;\langle q, v\rangle \leq \mathrm{D}_{C} g(x ; v), \forall v \in \mathbb{R}^{n}\right\} .
$$

By $\mathscr{P}\left(\mathbb{R}^{n}\right)$ we denote the family of all subsets of $\mathbb{R}^{n}$.

Corresponding to each type of tangent cones, say $\tau_{x} X$, one may introduce (e.g., [1]) a set-valued directional derivative of a multifunction $G(\cdot): X \subset \mathbb{R}^{n} \rightarrow \mathscr{P}\left(\mathbb{R}^{n}\right)$ (in particular of a single-valued mapping) at a point $(x, y) \in \operatorname{Graph}(G)$ as follows:

$$
\tau_{y} G(x ; v)=\left\{w \in \mathbb{R}^{n} ;(v, w) \in \tau_{(x, y)} \operatorname{Graph}(G)\right\}, \quad v \in \tau_{x} X .
$$


We recall that a set-valued map, $A(\cdot): \mathbb{R}^{n} \rightarrow \mathscr{P}\left(\mathbb{R}^{n}\right)$, is said to be a convex (resp., closed convex) process if $\operatorname{Graph}(A(\cdot)) \subset \mathbb{R}^{n} \times \mathbb{R}^{n}$ is a convex (resp., closed convex) cone. For the basic properties of convex processes we refer to [1], but we will use here only the above definition.

In what follows we are concerned with the discrete inclusion

$$
x_{i j} \in F_{i j}\left(x_{i j-1}, x_{i-1 j}, x_{i-1 j-1}\right), \quad i=0,1, \ldots, N, j=0,1, \ldots, N,
$$

where $F_{i j}(\cdot): \mathbb{R}^{3 n} \rightarrow \mathscr{P}\left(\mathbb{R}^{n}\right), i, j=0,1, \ldots, N . \mathscr{P}\left(\mathbb{R}^{n}\right)$ denotes the family of all subsets of $\mathbb{R}^{n}$.

Denote by $S_{F}$ the solution set of inclusion (2.15), that is

$$
\begin{aligned}
& S_{F}:=\left\{x=\left(x_{0}, x_{1}, \ldots, x_{N}\right) ; x_{i}=\left(x_{i 0}, x_{i 1}, \ldots, x_{i N}\right), x_{i j} \in \mathbb{R}^{n},\right. \\
&\left.i, j=0,1, \ldots, N, x \text { is a solution of }(2.15), x_{i j}=0, \text { if } i<0 \text { or } j<0\right\},
\end{aligned}
$$

and by $R_{F}^{N}:=\left\{x_{N N} ; x \in S_{F}\right\}$ the reachable set of inclusion (2.6).

We consider $\bar{x}=\left(\bar{x}_{0}, \bar{x}_{1}, \ldots, \bar{x}_{N}\right) \in S_{F}$ a solution of $(2.15)$.

In the sequel we will assume the following hypothesis.

Hypothesis 2.3. The set-valued maps $F_{i j}(\cdot)$ have nonempty compact convex values for all $i, j \in\{0,1, \ldots, N\}$ and there exists $L>0$ such that $F_{i j}(\cdot)$ is Lipschitz with the Lipschitz constant $L$, for all $i, j \in\{0,1, \ldots, N\}$.

In order to associate the linearized (variational) inclusion to our problem we need the following hypothesis.

Hypothesis 2.4. There exists $A_{i j}(\cdot): \mathbb{R}^{3 n} \rightarrow \mathscr{P}\left(\mathbb{R}^{n}\right), i, j=0,1, \ldots, N$ as a family of closed convex processes such that

$$
A_{i j}(u, v, w) \subset Q_{\bar{x}_{i j}} F_{i j}\left(\left(\bar{x}_{i j-1}, \bar{x}_{i-1 j}, \bar{x}_{i-1 j-1}\right) ;(u, v, w)\right),
$$

for all $u, v, w \in \mathbb{R}^{n}$, for all $i, j \in\{0,1, \ldots, N\}$.

Let $A_{0}$ be a derived cone to $F_{00}(0,0,0)$ at $\bar{x}_{00}$. To the problem $(2.15)$ we associate the linearized problem

$$
w_{i j} \in A_{i j}\left(w_{i j-1}, w_{i-1 j}, w_{i-1 j-1}\right), \quad w_{00} \in A_{0}, i, j=0,1, \ldots, N, i+j>0,
$$

with the boundary conditions $w_{i j}=0$, for $i<0$ or $j<0$.

Denote by $S_{A}$ the solution set of inclusion (2.18) and by $R_{A}^{N}$ the reachable set of inclusion (2.18).

We recall that if $A: \mathbb{R}^{n} \rightarrow \mathscr{P}\left(\mathbb{R}^{m}\right)$ is a set-valued map, then the adjoint of $A$ is the multifunction $A^{*}: \mathbb{R}^{m} \rightarrow \mathscr{P}\left(\mathbb{R}^{n}\right)$ defined by

$$
A^{*}(p)=\left\{q \in \mathbb{R}^{n} ;\langle q, v\rangle \leq\left\langle p, v^{\prime}\right\rangle, \forall\left(v, v^{\prime}\right) \in \operatorname{graph} A(\cdot)\right\}
$$


In the study of our optimization problem we need the next duality result.

Lemma 2.5 [12]. Assume that Hypothesis 2.4 is verified and let $r(\cdot): \mathbb{R}^{n} \rightarrow \mathscr{P}\left(\mathbb{R}^{n}\right)$ be the set-valued map defined by

$$
r(\alpha):=\left\{w_{N N} ; w=\left(w_{0}, \ldots, w_{N}\right) \text { is a solution of }(2.18), w_{00}=\alpha\right\} .
$$

Then, for all $b \in \mathbb{R}^{n}$,

$$
\begin{aligned}
& r^{*}(b)=\left\{u_{01}^{1}+u_{10}^{2}+u_{11}^{3} ;\left(u_{i j}^{1}, u_{i j}^{2}, u_{i j}^{3}\right) \in A_{i j}^{*}\left(u_{i j+1}^{1}+u_{i+1 j}^{2}+u_{i+1 j+1}^{3}\right),\right. \\
&\left.i, j=0,1, \ldots, N, 0<i+j<2 N,\left(u_{N N}^{1}, u_{N N}^{2}, u_{N N}^{3}\right) \in A_{N N}^{*}(b)\right\} .
\end{aligned}
$$

Corollary 2.6. Assume that Hypothesis 2.4 is satisfied and $A_{0} \subset Q_{\bar{x}_{00}} F_{00}(0,0,0)$ is a closed convex cone.

Then

$$
\begin{gathered}
\left(R_{A}^{N}\right)^{+} \subset\left\{q \in \mathbb{R}^{n} ; \exists u_{i j}^{1}, u_{i j}^{2}, u_{i j}^{3} \in \mathbb{R}^{n} \text { such that }\left(u_{i j}^{1}, u_{i j}^{2}, u_{i j}^{3}\right) \in A_{i j}^{*}\left(u_{i j+1}^{1}+u_{i+1 j}^{2}+u_{i+1 j+1}^{3}\right),\right. \\
\quad i, j=0,1, \ldots, N, 0<i+j<2 N,\left(u_{N N}^{1}, u_{N N}^{2}, u_{N N}^{3}\right) \in A_{N N}^{*}(q), \\
\left.u_{01}^{1}+u_{10}^{2}+u_{11}^{3} \in A_{0}^{+}\right\} .
\end{gathered}
$$

Proof. Obviously, $R_{A}^{N}=r\left(A_{0}\right)$, where $r(\cdot)$ was defined in the statement of Lemma 2.5. Therefore $\left(R_{A}^{N}\right)^{+}=\left(r\left(A_{0}\right)\right)^{+}=r^{*-1}\left(A_{0}^{+}\right)$. If $b \in\left(R_{A}^{N}\right)^{+}$, it follows that $r^{*}(b) \in A_{0}^{+}$and the corollary follows now from Lemma 2.5.

Finally, we recall the definition of local controllability.

Definition 2.7. Inclusion (2.15) is said to be locally controllable around the solution $\bar{x}$ if $\bar{x}_{N N} \in \operatorname{int}\left(R_{F}^{N}\right)$.

\section{The main result}

We prove that the reachable set $R_{A}^{N}$ of the variational multiparameter inclusion (2.18) is a derived cone to the reachable set $R_{F}^{N}$ at $\bar{x}_{N N}$.

Theorem 3.1. Let $A_{0} \subset \mathbb{R}^{n}$ be a derived cone to $F_{00}(0,0,0)$ and assume that Hypotheses 2.3 and 2.4 are satisfied.

Then the reachable set $R_{A}^{N}$ is a derived cone to $R_{F}^{N}$ at $\bar{x}_{N N}$.

Proof. In view of Definition 2.1, let $\left\{w_{N N}^{1}, \ldots, w_{N N}^{m}\right\} \subset R_{A}^{N}$, hence there exist the solutions $w^{1}=\left(w_{0}^{1}, w_{1}^{1}, \ldots, w_{N}^{1}\right), \ldots, w^{m}=\left(w_{0}^{m}, w_{1}^{m}, \ldots, w_{N}^{m}\right)$ to the variational inclusion $(2.18)$.

Since $A_{0} \subset \mathbb{R}^{n}$ is a derived cone to $F_{00}(0,0,0)$, there exists $\theta_{0}>0$ and continuous mappings $a_{0}(\cdot): S=\left[0, \theta_{0}\right]^{m} \rightarrow F_{00}(0,0,0)$ such that

$$
a_{0}(0)=\bar{x}_{00}, \quad \mathrm{D} a_{0}(0) s=\sum_{j=1}^{m} s_{j} w_{00}^{j}, \quad \forall s \in \mathbb{R}_{+}^{m} .
$$


Further on, for any $s=\left(s_{1}, \ldots, s_{m}\right) \in S$ we denote

$$
u(s)=\sum_{j=1}^{m} s_{j} w^{j}, \quad u(s)=\left(u_{0}(s), u_{1}(s), \ldots, u_{N}(s)\right), \quad y(s)=\bar{x}+u(s) .
$$

For $x=\left(x_{0}, x_{1}, \ldots, x_{N}\right) \in \mathbb{R}^{(N+1) n} \times \mathbb{R}^{(N+1) n} \times \cdots \mathbb{R}^{(N+1) n}$ we define

$$
F(x)=\left(F_{0}(x), F_{1}(x), \ldots, F_{N}(x)\right), \quad A(x)=\left(A_{0}(x), A_{1}(x), \ldots, A_{N}(x)\right),
$$

with

$$
F_{i}(x)=\left(\widetilde{F}_{i 0}(x), \widetilde{F}_{i 1}(x), \ldots, \widetilde{F}_{i N}(x)\right), \quad A_{i}(x)=\left(\widetilde{A}_{i 0}(x), \widetilde{A}_{i 1}(x), \ldots, \widetilde{A}_{i N}(x)\right),
$$

where

$$
\tilde{F}_{i j}(x):=F_{i j}\left(x_{i j-1}, x_{i-1 j}, x_{i-1 j-1}\right), \quad \tilde{A}_{i j}(x):=A_{i j}\left(x_{i j-1}, x_{i-1 j}, x_{i-1 j-1}\right),
$$

$i, j=0,1, \ldots, N, x_{i j}=0$ for $i<0$ or $j<0$ and $A_{00}(\cdot)=A_{0}$.

We prove next that

$$
\lim _{s \rightarrow 0} \frac{\mathrm{d}(y(s), F(y(s)))}{\|s\|}=0 .
$$

Since $A_{i j}(\cdot)$ are convex process, for any $s \in S \backslash\{0\}$ one has

$$
\begin{aligned}
u_{i j}\left(\frac{s}{\|s\|}\right) & \in A_{i j}\left(u_{i j-1}\left(\frac{s}{\|s\|}\right), u_{i-1 j}\left(\frac{s}{\|s\|}\right), u_{i-1 j-1}\left(\frac{s}{\|s\|}\right)\right) \\
& \subset Q_{\bar{x}_{i j}} F_{i j}\left(\left(\bar{x}_{i j-1}, \bar{x}_{i-1 j}, \bar{x}_{i-1 j-1}\right) ;\left(u_{i j-1}\left(\frac{s}{\|s\|}\right), u_{i-1 j}\left(\frac{s}{\|s\|}\right), u_{i-1 j-1}\left(\frac{s}{\|s\|}\right)\right)\right),
\end{aligned}
$$

and it follows from the definition of quasitangent derivative of lipschitzian set-valued maps that

$$
\begin{aligned}
\lim _{h \rightarrow 0+} \frac{1}{h} \mathrm{~d}\left(\bar{x}_{i j}+h u_{i j}\left(\frac{s}{\|s\|}\right), F_{i j}(\right. & \bar{x}_{i j-1}+h u_{i j-1}\left(\frac{s}{\|s\|}\right), \bar{x}_{i-1 j} \\
& \left.\left.+h u_{i-1 j}\left(\frac{s}{\|s\|}\right), \bar{x}_{i-1 j-1}+h u_{i-1 j-1}\left(\frac{s}{\|s\|}\right)\right)\right)=0 .
\end{aligned}
$$

In order to prove that (3.8) implies (3.6), we consider the compact metric space $S_{+}^{m-1}=$ $\left\{\sigma \in \mathbb{R}_{+}^{m} ;\|\sigma\|=1\right\}$ and the real functions $\phi_{i j}(\cdot, \cdot):\left(0, \theta_{0}\right] \times S_{+}^{m-1} \rightarrow \mathbb{R}_{+}$defined by $\phi_{i j}(\theta, \sigma):=\frac{1}{\theta} \mathrm{d}\left(\bar{x}_{i j}+\theta u_{i j}(\sigma), F_{i j}\left(\bar{x}_{i j-1}+\theta u_{i j-1}(\sigma), \bar{x}_{i-1 j}+\theta u_{i-1 j}(\sigma), \bar{x}_{i-1 j-1}+\theta u_{i-1 j-1}(\sigma)\right)\right)$,

which according to (3.8) has the property

$$
\lim _{\theta \rightarrow 0+} \phi_{i j}(\theta, \sigma)=0, \quad \forall \sigma \in S_{+}^{m-1}, t=0, \ldots, N-1 .
$$


Using the fact that $\phi_{i j}(\theta, \cdot)$ is Lipschitzian and the fact that $S_{+}^{m-1}$ is a compact metric space, from (3.10) it follows easily (e.g., [7, Proposition 4.4]) that

$$
\lim _{\theta \rightarrow 0+} \max _{\sigma \in S_{+}^{m-1}} \phi_{i j}(\theta, \sigma)=0
$$

which implies the fact that $\lim _{s \rightarrow 0} \phi_{i}(\|s\|, s /\|s\|)=0$. Therefore, $\lim _{s \rightarrow 0} \phi(\|s\|, s /\|s\|)=0$, where $\phi(\cdot)=\left(\phi_{0}(\cdot), \ldots, \phi_{N}(\cdot)\right), \phi_{i}(\cdot)=\left(\phi_{i 0}(\cdot), \ldots, \phi_{i N}(\cdot)\right)$ and using (3.1) it follows (3.6).

From the compactness of $F_{00}(0,0,0)$ and the fact that the values of $F_{i j}(\cdot, \cdot, \cdot)$ are compact, there exists a mapping $r(\cdot)=\left(r_{0}(\cdot), r_{1}(\cdot), \ldots, r_{N}(\cdot)\right): \mathbb{R}^{(N+1) n} \times \cdots \times \mathbb{R}^{(N+1) n} \rightarrow S_{F}$, $r_{i}(\cdot)=\left(r_{i 0}(\cdot), \ldots, r_{i N}(\cdot)\right), r_{i j}(\cdot)=0$ if $i<0$ or $j<0$ satisfying

$$
\begin{gathered}
\left\|x_{00}-r_{00}(x)\right\|=\mathrm{d}\left(x_{00}, F_{00}(0,0,0)\right), \\
\left\|x_{i j}-r_{i j}(x)\right\|=\mathrm{d}\left(x_{i j}, F_{i j}\left(r_{i j-1}(x), r_{i-1 j}(x), r_{i-1 j-1}(x)\right)\right), \quad i, j=0,1, \ldots, N .
\end{gathered}
$$

Moreover, by convexity of the values of $F_{i j}(\cdot, \cdot)$, the mapping $r(\cdot)$ is continuous.

On the other hand, we have

$$
\begin{aligned}
\left\|x_{i j}-r_{i j}(x)\right\|= & \mathrm{d}\left(x_{i j}, F_{i j}\left(r_{i j-1}(x), r_{i-1 j}(x), r_{i-1 j-1}(x)\right)\right) \leq \mathrm{d}\left(x_{i j}, F_{i j}\left(x_{i j-1}, x_{i-1 j}, x_{i-1 j-1}\right)\right) \\
& +L\left[\left\|x_{i j-1}-r_{i j-1}(x)\right\|+\left\|x_{i-1 j}-r_{i-1 j}(x)\right\|+\left\|x_{i-1 j-1}-r_{i-1 j-1}(x)\right\|\right] .
\end{aligned}
$$

Therefore, there exists $l>0$ depending only on $L$ such that

$$
\left\|x_{N N}-r_{N N}(x)\right\| \leq l \mathrm{~d}(x, F(x)), \quad \forall x \in \mathbb{R}^{(N+1)^{2 n}} .
$$

Finally, we define the mapping $a(\cdot): S \rightarrow R_{F}^{N}$ by

$$
a(s)=(r \circ y)_{N N}(s)
$$

Obviously, $a(\cdot)$ is continuous on $S$ and satisfies $a(0)=\bar{x}_{N N}$.

To end the proof we need to show that $a(\cdot)$ is differentiable at $s_{0}=0 \in S$ and its derivative is given by

$$
\operatorname{Da}(0)(s)=\sum_{j=1}^{m} s_{j} w_{N N}^{j}, \quad \forall s \in \mathbb{R}_{+}^{m},
$$

which is equivalent with the fact that

$$
\lim _{s \rightarrow 0} \frac{1}{\|s\|}\left(\left\|a(s)-a(0)-\sum_{j=1}^{m} s_{j} w_{N N}^{j}\right\|\right)=0 .
$$


One may write

$$
\begin{aligned}
\frac{1}{\|s\|}\left\|a(s)-a(0)-\sum_{j=1}^{m} s_{j} w_{N N}^{j}\right\| & \leq \frac{1}{\| s \mid}\left\|(r \circ y)_{N N}(s)-y_{N N}(s)\right\| \\
& \leq \frac{l}{\|s\|} \mathrm{d}(y(s), F(y(s))) .
\end{aligned}
$$

\section{Applications}

An important application of Theorem 3.1 concerns the local controllability of the discrete inclusion (2.15) in the sense of Definition 2.7.

Apart from Theorem 3.1 characterizing a derived cone to the reachable set, the main tool in the study of controllability is the remarkable property ([8, Theorem 4.7.4]) of the derived cones, according to which $x \in \operatorname{int}(X)$ if and only if $C=\mathbb{R}^{n}$ is a derived cone to $X$ at $x$.

Therefore, a straightforward application of this result and of Theorem 3.1 gives the following result.

Theorem 4.1. Let $\bar{x}, F_{i j}(\cdot), A_{0} \subset \mathbb{R}^{n}, A_{i j}(\cdot), i, j=0, \ldots, N$ satisfy the assumptions of Theorem 3.1. If the linearized inclusion (2.18) is controllable at $x_{N N} \in \mathbb{R}^{n}$ in the sense that $R_{A}^{N}=\mathbb{R}^{n}$, then the discrete inclusion (2.15) is locally controllable around the solution $\bar{x}$.

Consider now the problem

$$
\text { minimize } g\left(x_{N N}\right)
$$

over the solutions of the multiparameter discrete inclusion:

$$
x_{i j} \in F_{i j}\left(x_{i j-1}, x_{i-1 j}, x_{i-1 j-1}\right), \quad i=0,1, \ldots, N, j=0,1, \ldots, N,
$$

with endpoint constraints of the form

$$
x_{N N} \in X_{N}
$$

where $F_{i j}(\cdot): \mathbb{R}^{3 n} \rightarrow \mathscr{P}\left(\mathbb{R}^{n}\right), i, j=0, \ldots, N$, are given set-valued maps, $X_{N} \subset \mathbb{R}^{n}$, and $g(\cdot)$ : $\mathbb{R}^{n} \rightarrow \mathbb{R}$ is also a given function.

In what follows we obtain necessary optimality conditions for a solution $\bar{x}=\left(\bar{x}_{0}, \bar{x}_{1}, \ldots\right.$, $\left.\bar{x}_{N}\right)$ to the problem (4.1)-(4.3) in the form of minimum principle. The proof of maximum principle is due, mainly to the "intersection property" of derived cones obtained by Mirică (Lemma 2.2 above). A last step uses the Tuan and Ishizuka duality results in [12], that characterize the positive dual of the solution set of the variational inclusion associated to (4.2) in terms of the adjoint inclusion.

Theorem 4.2. Let $X_{N} \subset \mathbb{R}^{n}$ be a closed set, let $\bar{x} \in S_{F}$ be an optimal solution for problem (4.1)-(4.3) such that Hypothesis 2.4 is satisfied, and let $g(\cdot): \mathbb{R}^{n} \rightarrow \mathbb{R}$ be a locally Lipschitz function. 
Then for any derived cones $A_{0}$ of $F_{00}(0,0,0)$ at $\bar{x}_{00}$ and $C_{1}$ of $X_{N}$ at $\bar{x}_{N N}$, there exist $\lambda \in\{0,1\}, q \in \mathbb{R}^{n}$, and $u_{i j}^{1}, u_{i j}^{2}, u_{i j}^{3} \in \mathbb{R}^{n}$ such that

$$
\begin{gathered}
\left(u_{i j}^{1}, u_{i j}^{2}, u_{i j}^{3}\right) \in A_{i j}^{*}\left(u_{i j+1}^{1}+u_{i+1 j}^{2}+u_{i+1 j+1}^{3}\right), \quad 0<i+j<2 N \\
\left(u_{N N}^{1}, u_{N N}^{2}, u_{N N}^{3}\right) \in A_{N N}^{*}(q), \\
q \in \lambda \partial_{C} g\left(\bar{x}_{N N}\right)-C_{1}^{+}, \quad u_{01}^{1}+u_{10}^{2}+u_{11}^{3} \in A_{0}^{+}, \\
\left\langle u_{i j+1}^{1}+u_{i+1 j}^{2}+u_{i+1 j+1}^{3}, \bar{x}_{i j}\right\rangle=\min \left\{\left\langle u_{i j+1}^{1}+u_{i+1 j}^{2}+u_{i+1 j+1}^{3}, v\right\rangle ; v \in F_{i j}\left(\bar{x}_{i j-1}, \bar{x}_{i-1 j}, \bar{x}_{i-1 j-1}\right)\right\} \\
0<i+j<2 N \\
\left\langle q, \bar{x}_{N N}\right\rangle=\min \left\{\langle q, v\rangle ; v \in F_{N N}\left(\bar{x}_{N N-1}, \bar{x}_{N-1 N}, \bar{x}_{N-1 N-1}\right)\right\} \\
\left\langle u_{01}^{1}+u_{10}^{2}+u_{11}^{3}, \bar{x}_{00}\right\rangle=\min \left\{\left\langle v, \bar{x}_{00}\right\rangle ; v \in F_{00}(0,0,0)\right\} \\
\lambda+\|q\|>0 .
\end{gathered}
$$

Proof. We have $g\left(\bar{x}_{N N}\right)=\min \left\{g(x): x \in X_{N} \cap R_{F}^{N}\right\}$ and from definitions it follows that

$$
\mathrm{D}_{C} g\left(\bar{x}_{N N} ; v\right) \geq \overline{\mathrm{D}}_{K} g\left(\bar{x}_{N N} ; v\right) \geq 0, \quad \forall v \in K_{\bar{x}_{N N}}\left(X_{N} \cap R_{F}^{N}\right) .
$$

For all $i, j=0,1, \ldots, N$ and $y \in \mathbb{R}^{n}$ define

$$
\widetilde{A}_{i j}(u, v, w)=\mathrm{cl}\left(A_{i j}(u, v, w)+\bigcup_{t>0} \frac{1}{t}\left[F_{i j}\left(\bar{x}_{i j-1}, \bar{x}_{i-1 j}, \bar{x}_{i-1 j-1}\right)-\bar{x}_{i j}\right]\right) .
$$

Then, by [9, Proposition 3.5], $\left\{\widetilde{A}_{i j}\right\}_{i, j=0,1, \ldots, N}$ is a family of closed convex processes satisfying Hypothesis $2.4, A_{i j} \subset \tilde{A}_{i j}$, and moreover

$$
\tilde{A}_{i j}^{*}(y)= \begin{cases}A_{i j}^{*}(y), & \text { if }\left\langle y, \bar{x}_{i j}\right\rangle=\min \left\{\langle y, v\rangle ; v \in F_{i j}\left(\bar{x}_{i j-1}, \bar{x}_{i-1 j}, \bar{x}_{i-1 j-1}\right)\right\}, \\ \varnothing, & \text { otherwise. }\end{cases}
$$

From Theorem 3.1, $C^{N}:=R_{\widetilde{A}}^{N}$ is a derived cone to $X_{N}$ at $\bar{x}_{N N}$. We have two cases.

In the case when $C^{N}$ and $C_{1}$ are separable, there exists $q \in \mathbb{R}^{n} \backslash\{0\}$ such that

$$
\langle q, v\rangle \leq 0 \leq\langle q, w\rangle, \quad \forall v \in C_{1}, w \in C^{N},
$$

hence $q \in-\left(C_{1}\right)^{+}$and $q \in\left(C^{N}\right)^{+}$. According to Corollary 2.6 and (4.10) there exist $u_{i j}^{1}$, $u_{i j}^{2}, u_{i j}^{3} \in \mathbb{R}^{n}$ such that $u_{01}^{1}+u_{10}^{2}+u_{11}^{3} \in A_{0}^{+}$and (4.4), (4.6) hold true. Therefore, if we take $\lambda=0$ then (4.4)-(4.7) are verified.

In the case when $C^{N}$ and $C_{1}$ are not separable, we have $\left(C^{N} \cap C_{1}\right)^{+}=\left(C^{N}\right)^{+}+\left(C_{1}\right)^{+}$. It follows from Lemma 2.2 that $C^{N} \cap C_{1} \subset Q_{\bar{x}(N)}\left(X_{N} \cap R_{F}^{N}\right)$. From a simple separation result (e.g., [11, Lemma 5.1]), from the definition of Clarke's generalized gradient, and from (4.8), we obtain the existence of $q \in \partial_{C} g(\bar{x}(N)) \cap\left(\left(C^{N}\right)^{+}+\left(C_{1}\right)^{+}\right)$. Hence there exist $q_{1} \in\left(C^{N}\right)^{+}, q_{2} \in\left(C_{1}\right)^{+}$such that $q=q_{1}+q_{2}$. As in the first case, using Corollary 2.6 we 
deduce the existence of $u_{i j}^{1}, u_{i j}^{2}, u_{i j}^{3} \in \mathbb{R}^{n}$ such that $u_{01}^{1}+u_{10}^{2}+u_{11}^{3} \in A_{0}^{+}$and (4.4) holds. As in the first case, from (4.10) we obtain (4.6). We take in this case $\lambda=1$ and (4.7) is also verified.

In particular, when $F_{i j}(\cdot, \cdot, \cdot)$ are expressed in the parametrized form

$$
F_{i j}\left(x_{i j-1}, x_{i-1 j}, x_{i-1 j-1}\right)=f_{i j}\left(x_{i j-1}, x_{i-1 j}, x_{i-1 j-1}, U_{i j}\right),
$$

where $U_{i j} \subset \mathbb{R}^{m_{i j}}$ are compact sets for $0<i+j<2 N$ and $F_{00}(0,0,0)=\left\{\bar{x}_{00}\right\}$, for a solution $\bar{x}$ of inclusion (4.2) and its corresponding control $\bar{u}=\left(\bar{u}_{0}, \bar{u}_{1}, \ldots, \bar{u}_{N}\right), \bar{u}_{i}=\left(\bar{u}_{i 0}\right.$, $\left.\bar{u}_{i 1}, \ldots, \bar{u}_{i N}\right), \bar{u}_{i j} \in U_{i j}, i=0,1, \ldots, N, j=0,1, \ldots, N$, we define

$$
\begin{aligned}
A_{i j}^{1} & =\frac{\partial f_{i j}}{\partial x_{i j-1}}\left(\bar{x}_{i j-1}, \bar{x}_{i-1 j}, \bar{x}_{i-1 j-1}\right), \\
A_{i j}^{2} & =\frac{\partial f_{i j}}{\partial x_{i-1 j}}\left(\bar{x}_{i j-1}, \bar{x}_{i-1 j}, \bar{x}_{i-1 j-1}\right), \\
A_{i j}^{3} & =\frac{\partial f_{i j}}{\partial x_{i-1 j-1}}\left(\bar{x}_{i j-1}, \bar{x}_{i-1 j}, \bar{x}_{i-1 j-1}\right) .
\end{aligned}
$$

Hypothesis 4.3. For all $i, j \in\{0,1, \ldots, N\}$, the mappings $f_{i j}(\cdot, \cdot, \cdot)$ satisfy the following conditions.

(i) The function $f_{i j}\left(\cdot, \cdot, \cdot, u_{i j}\right)$ is Lipschitz for every fixed $u_{i j} \in U_{i j}$ and the function $f_{i j}\left(x_{i j-1}, x_{i-1 j}, x_{i-1 j-1}, \cdot\right)$ is continuous for every fixed $x_{i j-1}, x_{i-1 j}, x_{i-1 j-1}$.

(ii) The function $f_{i j}\left(\cdot, \cdot, \cdot, u_{i j}\right)$ is differentiable at $\left(x_{i j-1}, x_{i-1 j}, x_{i-1 j-1}\right)$.

Corollary 4.4. Let $\bar{x}=\left(\bar{x}_{0}, \bar{x}_{1}, \ldots, \bar{x}_{N}\right) \in S_{F}$ be an optimal solution for problem (4.1)-(4.2), with $F_{i j}$ defined by (4.12), such that Hypothesis 4.3 is satisfied. Consider $X_{N} \subset \mathbb{R}^{n}$ a closed set and $g(\cdot): \mathbb{R}^{n} \rightarrow \mathbb{R}$ a locally Lipschitz function.

Then for any derived cone $C_{1}$ of $X_{N}$ at $\bar{x}_{N N}$ there exist $q \in \mathbb{R}^{n}$ and a solution $p_{i j} \in \mathbb{R}^{n}$ such that

$$
\begin{gathered}
p_{i j}=\left(A_{i j+1}^{1}\right)^{*} p_{i j+1}+\left(A_{i+1 j}^{2}\right)^{*} p_{i+1 j}+\left(A_{i+1 j+1}^{3}\right)^{*} p_{i+1 j+1}, \quad 0<i+j<2 n, \\
p_{i j}=0, \quad \text { for } i>N \text { or } j>N, p_{N N} \in \lambda \partial_{C} g\left(\bar{x}_{N N}\right)-C_{1}^{+}, \\
\left\langle p_{i j}, \bar{x}_{i j}\right\rangle=\min \left\{\left\langle p_{i j}, v\right\rangle ; v \in F_{i j}\left(\bar{x}_{i j-1}, \bar{x}_{i-1 j}, \bar{x}_{i-1 j-1}\right)\right\}, \quad 0<i+j .
\end{gathered}
$$

Proof. We put $A_{i j}=\left(A_{i j}^{1}, A_{i j}^{2}, A_{i j}^{3}\right)$ and thus $A_{i j}$ satisfies Hypothesis 2.4. We apply Theorem 4.2 and we find $q \in \mathbb{R}^{n}$ and $u_{i j}^{1}, u_{i j}^{2}, u_{i j}^{3} \in \mathbb{R}^{n}$ such that

$$
\begin{gathered}
\left(u_{i j}^{1}, u_{i j}^{2}, u_{i j}^{3}\right)=A_{i j}^{*}\left(u_{i j+1}^{1}+u_{i+1 j}^{2}+u_{i+1 j+1}^{3}\right), \quad 0<i+j<2 N, \\
\left(u_{N N}^{1}, u_{N N}^{2}, u_{N N}^{3}\right)=A_{N N}^{a} s t(q),
\end{gathered}
$$

such that the minimum condition (4.6) holds.

It remains to put $p_{i j}:=u_{i j+1}^{1}+u_{i+1 j}^{2}+u_{i+1 j+1}^{3}$, where $u_{i j}^{1}=u_{i j}^{2}=u_{i j}^{3}=0$ if $i>N$ or $j>N$ and the proof is complete. 


\section{Multiparameter discrete inclusions}

\section{References}

[1] J.-P. Aubin and H. Frankowska, Set-Valued Analysis, Systems \& Control: Foundations \& Applications, vol. 2, Birkhäuser Boston, Massachusetts, 1990.

[2] V. G. Boltyanskiŭ, Optimal Control of Discrete Systems, Izdat. "Nauka”, Moscow, 1973.

[3] A. Cernea, Derived cones via relaxation for differential inclusions, Revue Roumaine de Mathématiques Pures et Appliquées 45 (2000), no. 1, 77-91.

[4] L Local controllability of hyperbolic differential inclusions via derived cones, Revue Roumaine de Mathématiques Pures et Appliquées 47 (2002), no. 1, 21-31.

[5] __ Controllability and maximum principle for discrete delay inclusions using derived cones, Revue Roumaine de Mathématiques Pures et Appliquées 50 (2005), no. 1, 19-29.

[6] Derived cones to reachable sets of discrete inclusions, submitted to Nonlinear Studies.

[7] A. Cernea and Ş. Mirică, Derived cones to reachable sets of differential inclusions, Mathematica (Cluj) 40 (63) (1998), no. 1, 35-62.

[8] M. R. Hestenes, Calculus of Variations and Optimal Control Theory, John Wiley \& Sons, New York, 1966.

[9] B. Kaśkosz, A maximum principle in relaxed controls, Nonlinear Analysis 14 (1990), no. 4, 357367.

[10] E. N. Makhmudov, Necessary conditions for problems of optimal control that are describable by differential inclusions with distributed parameters, Soviet Mathematics. Doklady 38 (1989), no. 3, 467-471.

[11] Ş. Mirică, New proof and some generalizations of the minimum principle in optimal control, Journal of Optimization Theory and Applications 74 (1992), no. 3, 487-508.

[12] H. D. Tuan and Y. Ishizuka, On controllability and maximum principle for discrete inclusions, Optimization 34 (1995), no. 4, 293-316.

Aurelian Cernea: Faculty of Mathematics and Informatics, University of Bucharest,

Street Academiei 14, 010014 Bucharest, Romania

E-mail addresses: acernea68@yahoo.com; acernea@math.math.unibuc.ro 


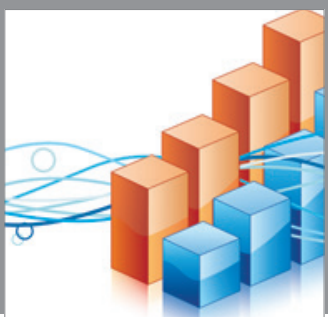

Advances in

Operations Research

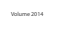

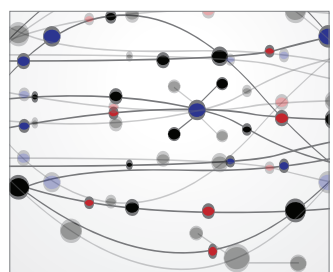

\section{The Scientific} World Journal
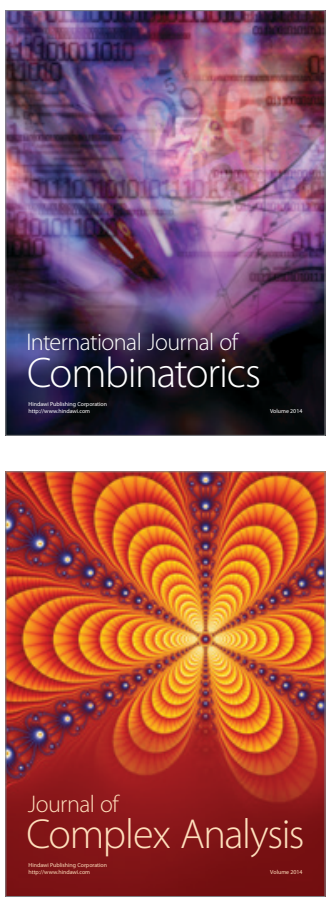

International Journal of

Mathematics and

Mathematical

Sciences
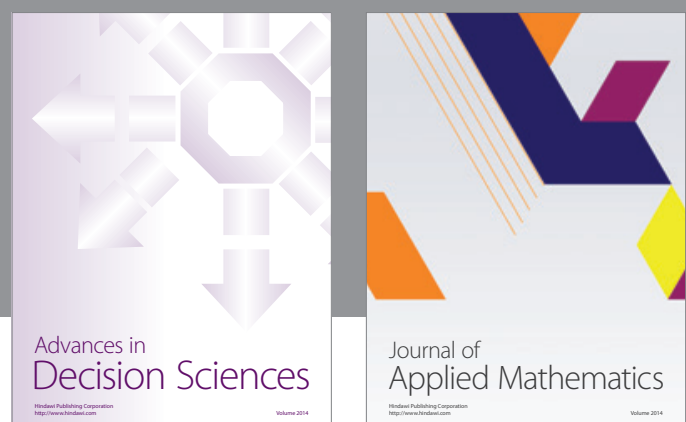

Journal of

Applied Mathematics
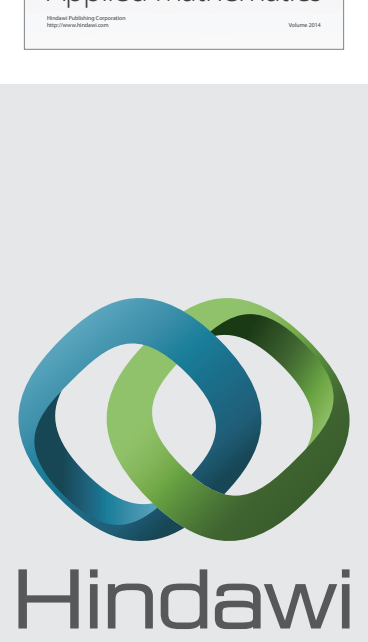

Submit your manuscripts at http://www.hindawi.com
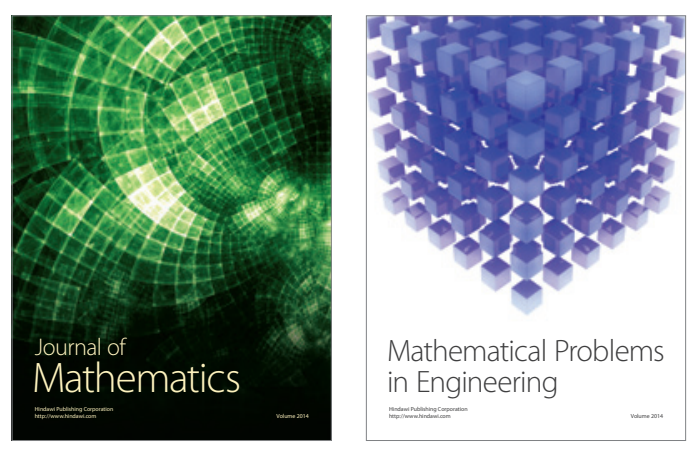

Mathematical Problems in Engineering
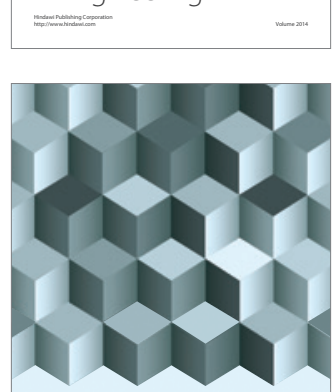

Journal of

Function Spaces
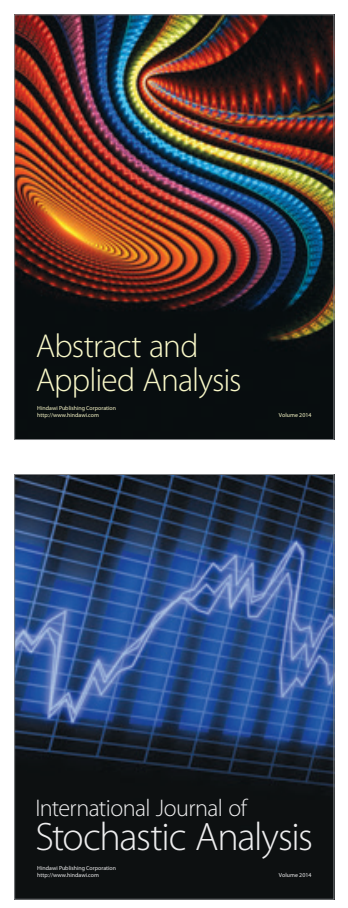

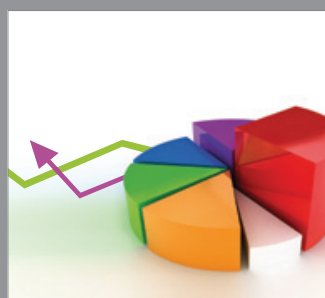

ournal of

Probability and Statistics

Promensencen
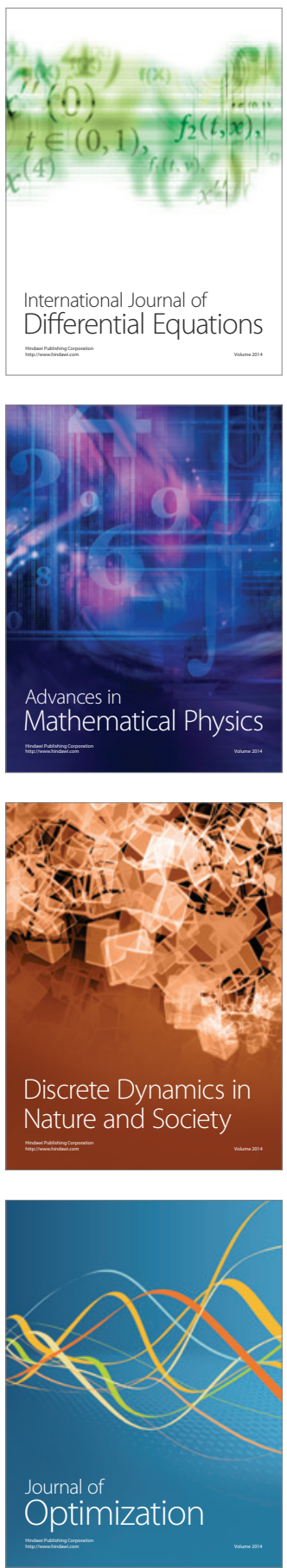